\title{
The use of LigaSure for preservation of a previous coronary artery bypass graft by using the left internal thoracic artery in a left upper lobectomy
}

\author{
Mario Santini, MD, Alfonso Fiorello, MD, Giovanni Vicidomini, MD, PhD, and Paolo Laperuta, MD, Naples, Italy
}

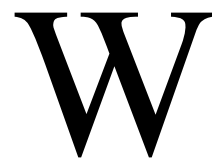

e report an interesting clinical case concerning the use of an electrothermal bipolar tissue-sealing system (LigaSure, Valleylab, Inc, Boulder, Colo) ${ }^{1}$ in a particularly challenging technical situation, such as the resection of the left upper pulmonary lobe in a patient with a previous coronary artery bypass graft $(\mathrm{CABG})$ with the left internal thoracic artery (LITA).

\begin{abstract}
Clinical Summary
In a 63-year-old man, an opacity localized in the left upper pulmonary lobe was incidentally discovered on chest radiographic analysis. He had undergone CABG 8 years before with the LITA on the left anterior descending coronary artery. Clinical and laboratory evaluation showed no abnormalities. Spirometric results were normal. Computed tomographic analysis demonstrated a peripheral mass in the left upper lobe, and there was no significant mediastinal adenopathy. Bronchoscopy did not demonstrate abnormalities of the left upper bronchus, and percutaneous needle biopsy showed a non-small cell lung cancer (NSCLC). A standard posterolateral thoracotomy incision was performed through the fifth intercostal space. A 6-cm tumor was found in the left upper lobe (Figure 1). The dissection of the lobe from the pericardium was possible, but dense adhesions were identified between the LITA and the apex of the upper lobe. The challenge was to do an upper lobectomy while avoiding injury to the LITA. Therefore the part of parenchyma adjacent to the LITA was separated from the upper lobe by using LigaSure system (Figure 2). After this maneuver, we performed an anatomic upper lobectomy, leaving a small strip of parenchyma adjacent to the LITA; we tested the arterial graft manually, and a good pulsation was found. Anatomopathologic studies showed a $6-\mathrm{cm}$ stage IB squamous cell carcinoma, according to TNM classification. ${ }^{2}$ The patient's postoperative course was unremarkable, and he was discharged on the seventh postoperative day.
\end{abstract}

\footnotetext{
From the Department of Thoracic Surgery, Second University of Naples, Naples, Italy.

Received for publication Nov 16, 2007; accepted for publication Dec 9, 2007.

Address for reprints: Mario Santini, MD, Chirurgia Toracica-Seconda Università di Napoli Piazza Miraglia, 2, I-80138 Naples, Italy (E-mail: mario. santini@unina2.it).

J Thorac Cardiovasc Surg 2008;136:222-3

$0022-5223 / \$ 34.00$

Copyright $\odot 2008$ by The American Association for Thoracic Surgery doi:10.1016/j.jtcvs.2007.12.049
}

\section{Discussion}

LITA harvesting for CABG often complicates subsequent thoracic procedures for the formation of adhesion in the left chest. In the future, thoracic surgery might be increasingly confronted with this particularly challenging technical situation because of the growing number of elderly patients having already undergone CABG and affected by potentially resectable lung cancer. The adhesions between graft and lung might complicate the exposition of the pulmonary hilum and make anatomic resection difficult. In the literature we found only 3 articles on this topic.

Singhatanadgige and colleagues ${ }^{3}$ illustrate the complete dissection of the anterior segment of the left upper pulmonary lobe from the LITA. Halkos and associates ${ }^{4}$ describe performing a left upper pulmonary lobectomy for NSCLC by dissecting the lung parenchyma around the LITA. The authors report leaving a small portion of lung parenchyma adherent to the LITA using multiple firings of an endo-gastrointestinal anastomosis (GIA) stapling device.

Green and Heitmiller ${ }^{5}$ report a patient with NSCLC of the left upper lobe involving a previous LITA graft. The coronary artery and LITA catheterization show that the myocardial graft is angiographically nonessential. Thus the left upper lobe is resected, with sacrifice of the LITA.

In our case the complete dissection of the left upper lobe from the LITA is associated with high risk of rupture of the LITA and potential acute myocardial ischemia. According to the surgical technique proposed by Halkos and associates, ${ }^{3}$ a small portion of parenchyma is left adherent to the LITA. However, compared with the use of a stapler, we prefer to use the LigaSure system for several reasons. The LigaSure allows us to delimit as much

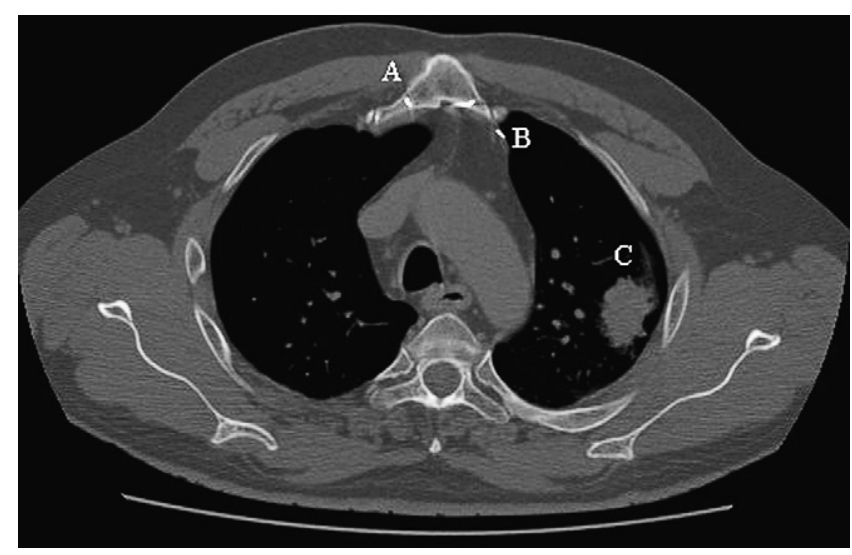

Figure 1. An axial computed tomographic image shows the closure of the median sternotomy $(A)$, the surgical clips adjacent to the LITA graft (B), and left upper lung cancer (C). 


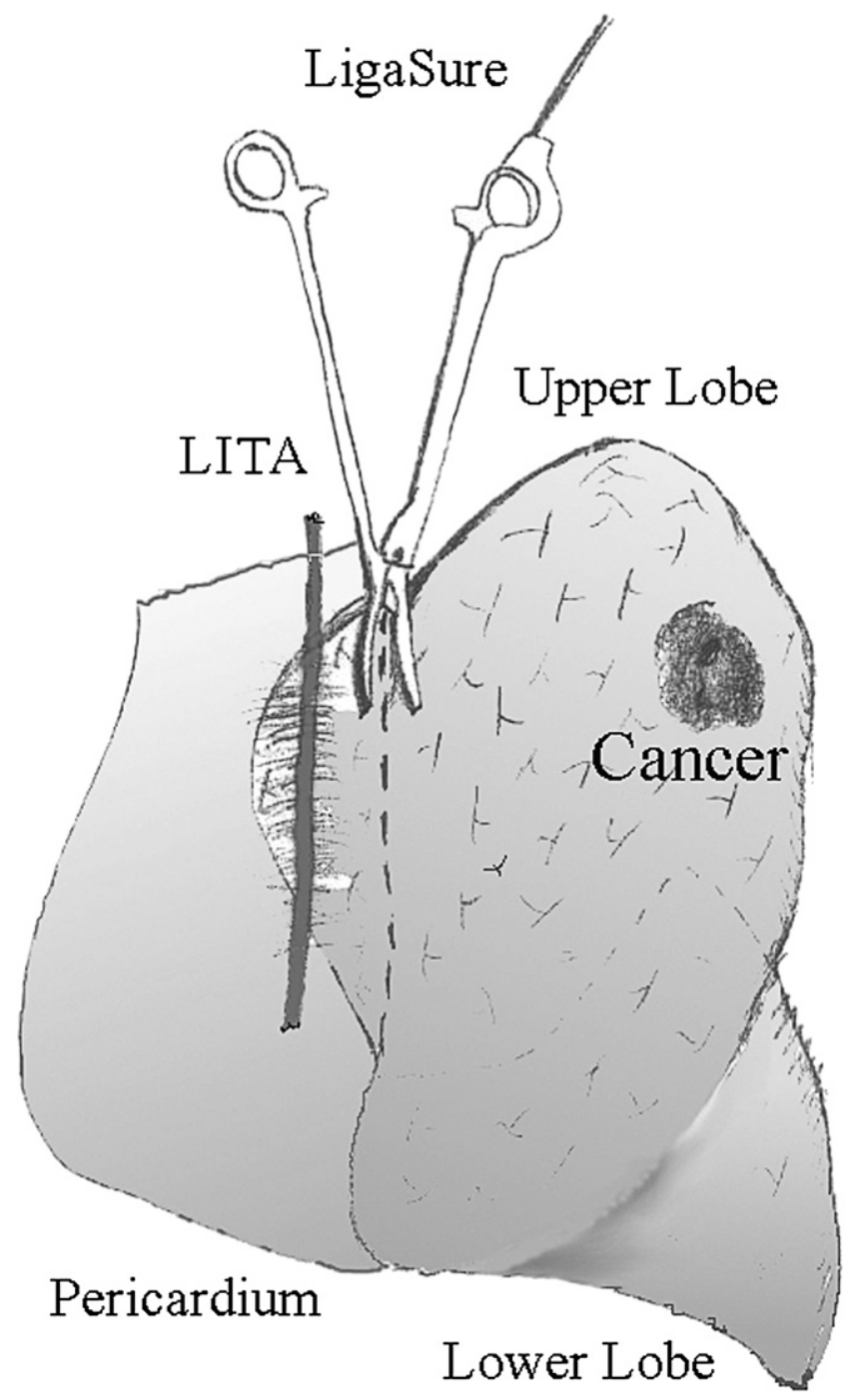

Figure 2. LigaSure allowed a better resection between the portion of parenchyma adherent to the left internal thoracic artery (LITA) to the rest of upper lobe. Furthermore, it permitted us to perform anatomic upper lobectomy and to leave a smaller part of lung with minimum possibility of cancer recurrence. as possible the resection between the LITA and the left upper lobe. Furthermore, it permits us to leave a strip of parenchyma adjacent to the LITA, with a minimal possibility of cancer recurrence. However, the small portion of the lung left in place is a nonventilated portion and theoretically it would not pose any problem, such as cyanosis or spilling of bronchial secretions. Finally, the Ligasure system allows a reduction of management cost because it does not use multiple firings.

In patients with a previous CABG with a LITA graft, a left upper lobectomy is a matter of thinking. In case of suspicion of LITA invasion, coronary and LITA catheterization should be added to the standard staging evaluation. If myocardial graft flow is shown to be angiographically nonessential, the tumor would be made amenable to complete resection by sacrificing the LITA graft, as proposed by Greene and Heitmiller. ${ }^{5}$ In other cases most care should be taken to preserve the CABG. The LigaSure system can be very useful in performing a safe dissection between the LITA and lung parenchyma, avoiding injury of the artery graft and safeguarding the hemodynamic condition of the patient.

\section{References}

1. Santini M, Vicidomini G, Baldi A, Gallo G, Laperuta P, Busiello L, et al. Use of an electrothermal bipolar tissue sealing system in lung surgery. Eur J Cardiothorac Surg. 2006;29:226-30.

2. Mountain CF. Revision in the International System for Staging Lung Cancer. Chest. 1997;111:1710-7.

3. Singhatanadgige S, Sindhavanada W, Kittayarak C. Left upper lobectomy after CABG with the left internal mammary artery graft. J Med Assoc Thai. 2006;89:887-9.

4. Halkos ME, Sherman AJ, Miller JI. Preservation of the LIMA pedicle after cardiac surgery in left upper lobectomy. Ann Thorac Surg. 2003;76:280-1.

5. Greene PS, Heitmiller RF. Lung cancer and the left internal mammary artery graft. Ann Thorac Surg. 1994;57:1029-30. 